\title{
Neutrosophic Soft Expert Sets
}

\author{
Mehmet Şahin'1, Shawkat Alkhazaleh², Vakkas Uluçay ${ }^{1}$ \\ ${ }^{1}$ Department of Mathematics, Gaziantep University, Gaziantep, Turkey \\ ${ }^{2}$ Department of Mathematics, Faculty of Science and Art, Shaqra University, Shaqra, KSA \\ Email: mesahin@gantep.edu.tr, shmk79@gmail.com, vulucay27@gmail.com
}

Received 14 November 2014; revised 6 December 2014; accepted 24 December 2014

Copyright (C 2015 by authors and Scientific Research Publishing Inc.

This work is licensed under the Creative Commons Attribution International License (CC BY). http://creativecommons.org/licenses/by/4.0/

(c) $\underset{\mathrm{EY}}{\mathrm{EY}}$ Open Access

\begin{abstract}
In this paper we introduce the concept of neutrosophic soft expert set (NSES). We also define its basic operations, namely complement, union, intersection, AND and OR, and study some of their properties. We give examples for these concepts. We give an application of this concept in a decision-making problem.
\end{abstract}

Keywords

Soft Expert Set, Neutrosophic Soft Set, Neutrosophic Soft Expert Set

\section{Introduction}

In some real-life problems in expert system, belief system, information fusion and so on, we must consider the truth-membership as well as the falsity-membership for proper description of an object in uncertain, ambiguous environment. Intuitionistic fuzzy sets were introduced by Atanassov [1]. After Atanassov's work, Smarandache [2] [3] introduced the concept of neutrosophic set which is a mathematical tool for handling problems involving imprecise, indeterminacy and inconsistent data. In 1999, Molodtsov [4] initiated a novel concept of soft set theory as a new mathematical tool for dealing with uncertainties. After Molodtsov's work, some different operations and applications of soft sets were studied by Chen et al. [5] and Maji et al. [6]. Later, Maji [7] firstly proposed neutrosophic soft sets with operations. Alkhazaleh et al. generalized the concept of fuzzy soft expert sets which include that possibility of each element in the universe is attached with the parameterization of fuzzy sets while defining a fuzzy soft expert set [8]. Alkhazaleh et al. [9] generalized the concept of parameterized intervalvalued fuzzy soft sets, where the mapping in which the approximate function are defined from fuzzy parameters set, and they gave an application of this concept in decision making. In the other study, Alkhazaleh and Salleh [10] introduced the concept soft expert sets where user can know the opinion of all expert sets. Alkhazaleh and Salleh [11] generalized the concept of a soft expert set to fuzzy soft expert set, which is a more effective and useful. They also defined its basic operations, namely complement, union, intersection, AND and OR, and gave 
an application of this concept in decision-making problem. They also studied a mapping on fuzzy soft expert classes and its properties. Our objective is to introduce the concept of neutrosophic soft expert set. In Section 1, we introduce from intuitionistic fuzzy sets to soft expert sets. In Section 2, preliminaries are given. In Section 3 , we also define the concept of neutrosophic soft expert set and its basic operations, namely complement, union, intersection AND and OR. In Section 4, we give an application of this concept in a decision-making problem. In Section 5 conclusions are given.

\section{Preliminaries}

In this section we recall some related definitions.

2.1. Definition: [3] Let $U$ be a space of points (objects), with a generic element in $U$ denoted by $u$. A neutrosophic set ( $N$-sets) $A$ in $U$ is characterized by a truth-membership function $T_{A}$, a indeterminacy-membership function $I_{A}$ and a falsity-membership function $F_{A} . T_{A}(u) ; I_{A}(u)$ and $F_{A}(u)$ are real standard or nonstandard subsets of $[0,1]$. It can be written as

$$
A=\left\{\left\langle u,\left(T_{A}(u), I_{A}(u), F_{A}(u)\right)\right\rangle: u \in U, T_{A}(u), I_{A}(u), F_{A}(u) \in[0,1]\right\} .
$$

There is no restriction on the sum of $T_{A}(u) ; I_{A}(u)$ and $F_{A}(u)$, so

$$
0 \leq \sup _{A}(u)+\sup I_{A}(u)+\sup _{A}(u) \leq 3 .
$$

2.2. Definition: [7] Let $U$ be an initial universe set and $E$ be a set of parameters. Consider $A \subseteq E$. Let $P(U)$ denotes the set of all neutrosophic sets of $U$. The collection $(F, A)$ is termed to be the soft neutrosophic set over $U$, where $F$ is a mapping given by $F: A \rightarrow P(U)$.

2.3. Definition: [6] A neutrosophic set $A$ is contained in another neutrosophic set $B$ i.e. $A \subseteq B$ if $\forall x \in X$, $T_{A}(x) \leq T_{B}(x), \quad I_{A}(x) \leq I_{B}(x), \quad F_{A}(x) \geq F_{B}(x)$.

Let $U$ be a universe, $E$ a set of parameters, and $X$ a soft experts (agents). Let $O$ be a set of opinion, $Z=E \times X \times O$ and $A \subseteq Z$.

2.4. Definition: [9] A pair $(F, A)$ is called a soft expert set over $U$, where $F$ is mapping given by $F: A \rightarrow P(U)$ where $P(U)$ denotes the power set of $U$.

2.5. Definition: [11] A pair $(F, A)$ is called a fuzzy soft expert set over $U$, where $F$ is mapping given by $F: A \rightarrow I^{U}$ where $I^{U}$ denotes the set of all fuzzy subsets of $U$.

2.6. Definition: [11] For two fuzzy soft expert sets $(F, A)$ and $(G, B)$ over $U,(F, A)$ is called a fuzzy soft expert subset of $(G, B)$ if

1) $B \subseteq A$,

2) $\forall \varepsilon \in A, F(\varepsilon)$ is fuzzy subset of $G(\varepsilon)$.

This relationship is denoted by $(F, A) \subseteq(G, B)$. In this case $(G, B)$ is called a fuzzy soft expert superset of $(F, A)$.

2.7. Definition: [11] Two fuzzy soft expert sets $(F, A)$ and $(G, B)$ over $U$ are said to be equal.

If $(F, A)$ is a fuzzy soft expert subset of $(G, B)$ and $(G, B)$ is a fuzzy soft expert subset of $(F, A)$.

2.8. Definition: [11] An agree-fuzzy soft expert set $(F, A)_{1}$ over $U$ is a fuzzy soft expert subset of $(F, A)$ defined as follow

$$
(F, A)_{1}=\left\{F_{1}(\alpha): \alpha \in E \times X \times\{1\}\right\} .
$$

2.9. Definition: [11] A disagree-fuzzy soft expert set $(F, A)_{0}$ over $U$ is a fuzzy soft expert subset of $(F, A)$ defined as follow

$$
(F, A)_{0}=\left\{F_{0}(\alpha): \alpha \in E \times X \times\{0\}\right\} .
$$

2.10. Definition: [11] Complement of a fuzzy soft expert set. The complement of a fuzzy soft expert set $(F, A)$ denoted by $(F, A)^{c}$ and is defined as $(F, A)^{c}=\left(F^{c}, \neg A\right)$ where $F^{c}=\neg A \rightarrow I^{U}$ is mapping given by

$$
F^{c}(\alpha)=c(F(\alpha)) \quad \forall \alpha \in A,
$$


where $c$ is a fuzzy complement.

2.11. Definition: [11] The intersection of fuzzy soft expert sets $(F, A)$ and $(G, B)$ over $U$, denoted by $(F, A) \tilde{\cap}(G, B)$, is the fuzzy soft expert set $(H, C)$ where $C=A \cup B$ and $\forall \varepsilon \in C$,

$$
H(\varepsilon)= \begin{cases}F(\varepsilon), & \text { if } \varepsilon \in A-B \\ G(\varepsilon), & \text { if } \varepsilon \in B-A \\ t(F(\varepsilon), G(\varepsilon)), & \text { if } \varepsilon \in A \cap B\end{cases}
$$

where $t$ is a $t$-norm.

2.12. Definition: [11] The intersection of fuzzy soft expert sets $(F, A)$ and $(G, B)$ over $U$, denoted by $(F, A) \tilde{\cup}(G, B)$, is the fuzzy soft expert set $(H, C)$ where $C=A \cup B$ and $\forall \varepsilon \in C$,

$$
H(\varepsilon)= \begin{cases}F(\varepsilon), & \text { if } \varepsilon \in A-B \\ G(\varepsilon), & \text { if } \varepsilon \in B-A \\ s(F(\varepsilon), G(\varepsilon)), & \text { if } \varepsilon \in A \cap B\end{cases}
$$

where $s$ is an s-norm.

2.13. Definition: [11] If $(F, A)$ and $(G, B)$ are two fuzzy soft expert sets over $U$ then " $(F, A)$ AND $(G, B)$ " denoted by $(F, A) \wedge(G, B)$ is defined by

$$
(F, A) \wedge(G, B)=(H, A \times B)
$$

such that $H(\alpha, \beta)=t(F(\alpha), G(\beta)), \forall(\alpha, \beta) \in A \times B$ where $t$ is a $t$-norm.

2.14. Definition: [11] If $(F, A)$ and $(G, B)$ are two fuzzy soft expert sets over $U$ then " $(F, A)$ OR $(G, B)$ " denoted by $(F, A) \vee(G, B)$ is defined by

$$
(F, A) \vee(G, B)=(H, A \times B)
$$

such that $H(\alpha, \beta)=s(F(\alpha), G(\beta)), \forall(\alpha, \beta) \in A \times B$ where $s$ is an $s$-norm.

Using the concept of neutrosophic set now we introduce the concept of neutrosophic soft expert set.

\section{Neutrosophic Soft Expert Set}

In this section, we introduce the definition of a neutrosophic soft expert set and give basic properties of this concept.

Let $U$ be a universe, $E$ a set of parameters, $X$ a set of experts (agents), and $O=\{1=$ agree, $0=$ disagree $\}$ a set of opinions. Let $Z=E \times X \times O$ and $A \subseteq Z$.

3.1. Definition: A pair $(F, A)$ is called a neutrosophic soft expert set over $U$, where $F$ is mapping given by

$$
F: A \rightarrow P(U)
$$

where $P(U)$ denotes the power neutrosophic set of $U$. For definition we consider an example.

3.1. Example: Suppose the following $U$ is the set of car under consideration $E$ is the set of parameters. Each parameter is a neutrosophic word or sentence involving neutrosophic words.

$$
\begin{aligned}
& U=\left\{u_{1}, u_{2}, u_{3}\right\} \\
& E=\{\text { easy to use; quality }\}=\left\{e_{1}, e_{2}\right\} \\
& X=\{p, q, r\}
\end{aligned}
$$

be a set of experts. Suppose that

$$
\begin{aligned}
& F\left(e_{1}, p, 1\right)=\left\{\left\langle u_{1}, 0.3,0.5,0.7\right\rangle,\left\langle u_{3}, 0.5,0.6,0.3\right\rangle\right\} \\
& F\left(e_{1}, q, 1\right)=\left\{\left\langle u_{2}, 0.8,0.2,0.3\right\rangle,\left\langle u_{3}, 0.9,0.5,0.7\right\rangle\right\}
\end{aligned}
$$




$$
\begin{aligned}
& F\left(e_{1}, r, 1\right)=\left\{\left\langle u_{1}, 0.4,0.7,0.6\right\rangle\right\} \\
& F\left(e_{2}, p, 1\right)=\left\{\left\langle u_{1}, 0.4,0.2,0.3\right\rangle,\left\langle u_{2}, 0.7,0.1,0.3\right\rangle\right\} \\
& F\left(e_{2}, q, 1\right)=\left\{\left\langle u_{3}, 0.3,0.4,0.2\right\rangle\right\} \\
& F\left(e_{2}, r, 1\right)=\left\{\left\langle u_{2}, 0.3,0.4,0.9\right\rangle\right\} \\
& F\left(e_{1}, p, 0\right)=\left\{\left\langle u_{2}, 0.5,0.2,0.3\right\rangle\right\} \\
& F\left(e_{1}, q, 0\right)=\left\{\left\langle u_{1}, 0.6,0.3,0.5\right\rangle\right\} \\
& F\left(e_{1}, r, 0\right)=\left\{\left\langle u_{2}, 0.7,0.6,0.4\right\rangle,\left\langle u_{3}, 0.9,0.5,0.7\right\rangle\right\} \\
& F\left(e_{2}, p, 0\right)=\left\{\left\langle u_{3}, 0.7,0.9,0.6\right\rangle\right\} \\
& F\left(e_{2}, q, 0\right)=\left\{\left\langle u_{1}, 0.7,0.3,0.6\right\rangle,\left\langle u_{2}, 0.6,0.2,0.5\right\rangle\right\} \\
& F\left(e_{2}, r, 0\right)=\left\{\left\langle u_{1}, 0.6,0.2,0.5\right\rangle,\left\langle u_{3}, 0.7,0.2,0.8\right\rangle\right\}
\end{aligned}
$$

The neutrosophic soft expert set $(F, Z)$ is a parameterized family $\left\{F\left(e_{i}\right), i=1,2,3, \cdots\right\}$ of all neutrosophic sets of $U$ and describes a collection of approximation of an object.

3.1. Definition: Let $(F, A)$ and $(G, B)$ be two neutrosophic soft expert sets over the common universe $U$. $(F, A)$ is said to be neutrosophic soft expert subset of $(G, B)$, if $A \tilde{\subset} B$ and $T_{F(e)}(x) \tilde{\leq} T_{G(e)}(x)$, $I_{F(e)}(x) \tilde{\leq} I_{G(e)}(x), \quad F_{F(e)}(x) \tilde{\geq} F_{G(e)}(x) \forall e \in A, x \in U$. We denote it by $(F, A) \tilde{\subseteq}(G, B)$.

$(F, A)$ is said to be neutrosophic soft expert superset of $(G, B)$ if $(G, B)$ is a neutrosophic soft expert subset of $(F, A)$. We denote by $(F, A) \tilde{\supseteq}(G, B)$.

3.2. Example: Suppose that a company produced new types of its products and wishes to take the opinion of some experts about concerning these products. Let $U=\left\{u_{1}, u_{2}, u_{3}\right\}$ be a set of product, $E=\left\{e_{1}, e_{2}\right\}$ a set of decision parameters where $e_{i}(i=1,2)$ denotes the decision "easy to use", "quality" respectively and let $X=\{p, q, r\}$ be a set of experts. Suppose

$$
\begin{aligned}
& A=\left\{\left(e_{1}, p, 1\right),\left(e_{2}, p, 0\right),\left(e_{1}, q, 1\right),\left(e_{1}, r, 0\right),\left(e_{2}, r, 1\right)\right\} \\
& B=\left\{\left(e_{1}, p, 1\right),\left(e_{2}, p, 0\right),\left(e_{1}, q, 1\right)\right\}
\end{aligned}
$$

Clearly $B \tilde{\subset} A$. Let $(F, A)$ and $(G, B)$ be defined as follows:

$$
\begin{aligned}
(F, A)=\left\{\left[\left(e_{1}, p, 1\right),\left\langle u_{1}, 0.3,0.5,0.7\right\rangle,\left\langle u_{2}, 0.5,0.2,0.3\right\rangle\right],\left[\left(e_{2}, p, 0\right),\left\langle u_{2}, 0.2,0.4,0.7\right\rangle\right],\right. & \\
& {\left[\left(e_{1}, q, 1\right),\left\langle u_{1}, 0.6,0.3,0.5\right\rangle,\left\langle u_{2}, 0.6,0.2,0.3\right\rangle\right],\left[\left(e_{1}, r, 0\right),\left\langle u_{1}, 0.2,0.7,0.3\right\rangle\right], } \\
& {\left.\left[\left(e_{2}, r, 1\right),\left\langle u_{2}, 0.3,0.4,0.9\right\rangle,\left\langle u_{3}, 0.7,0.2,0.8\right\rangle\right]\right\}, } \\
(G, B)=\left\{\left[\left(e_{1}, p, 1\right),\left\langle u_{1}, 0.3,0.5,0.7\right\rangle,\left\langle u_{2}, 0.5,0.2,0.3\right\rangle\right],\left[\left(e_{2}, p, 0\right),\left\langle u_{2}, 0.2,0.4,0.7\right\rangle\right],\right. & \\
& {\left.\left[\left(e_{1}, q, 1\right),\left\langle u_{1}, 0.6,0.3,0.5\right\rangle,\left\langle u_{2}, 0.6,0.2,0.3\right\rangle\right]\right\} . }
\end{aligned}
$$

Therefore

$$
(F, A) \tilde{\supseteq}(G, B) \text {. }
$$

3.3. Definition: Equality of two neutrosophic soft expert sets. Two (NSES), $(F, A)$ and $(G, B)$ over the common universe $U$ are said to be equal if $(F, A)$ is neutrosophic soft expert subset of $(G, B)$ and $(G, B)$ is neutrosophic soft expert subset of $(F, A)$. We denote it by 


$$
(F, A)=(G, B) .
$$

3.4. Definition: NOT set of set parameters. Let $E=\left\{e_{1}, e_{2}, \cdots, e_{n}\right\}$ be a set of parameters. The NOT set of $E$ is denoted by $\neg E=\left\{\neg e_{1}, \neg e_{2}, \cdots, \neg e_{n}\right\}$ where $\neg e_{i}=$ not $e_{i}, \forall i$.

3.3. Example: Consider 3.2.example. Here $\neg E=\{$ not easy to use, not quality $\}$.

3.5. Definition: Complement of a neutrosophic soft expert set. The complement of a neutrosophic soft expert set $(F, A)$ denoted by $(F, A)^{c}$ and is defined as $(F, A)^{c}=\left(F^{c}, \neg A\right)$ where $F^{c}=\neg A \rightarrow P(U)$ is mapping given by $F^{c}(x)=$ neutrosophic soft expert complement with $T_{F^{c}(x)}=F_{F(x)}, I_{F^{c}(x)}=I_{F(x)}, \quad F_{F^{c}(x)}=T_{F(x)}$.

3.4. Example: Consider the 3.1 Example. Then $(F, Z)^{c}$ describes the "not easy to use of the car" we have

$$
\begin{aligned}
&(F, Z)^{c}=\left\{\left(\neg e_{1}, p, 1\right),\left[\left\langle u_{2}, 0.3,0.2,0.5\right\rangle\right]\left[\left(\neg e_{1}, q, 1\right),\left\langle u_{1}, 0.5,0.3,0.6\right\rangle\right],\right. \\
& {\left[\left(\neg e_{1}, r, 1\right),\left\langle u_{2}, 0.4,0.6,0.7\right\rangle,\left\langle u_{3}, 0.7,0.5,0.9\right\rangle\right], } \\
& {\left[\left(\neg e_{2}, p, 1\right),\left\langle u_{3}, 0.6,0.9,0.7\right\rangle\right], } \\
& {\left[\left(\neg e_{2}, q, 1\right),\left\langle u_{1}, 0.6,0.3,0.7\right\rangle,\left\langle u_{2}, 0.5,0.2,0.6\right\rangle\right], } \\
& {\left[\left(\neg e_{2}, r, 1\right),\left\langle u_{1}, 0.5,0.2,0.6\right\rangle,\left\langle u_{3}, 0.8,0.2,0.7\right\rangle\right], } \\
& {\left[\left(\neg e_{1}, p, 0\right),\left\langle u_{1}, 0.7,0.5,0.3\right\rangle,\left\langle u_{3}, 0.3,0.6,0.5\right\rangle\right], } \\
& {\left[\left(\neg e_{1}, q, 0\right),\left\langle u_{2}, 03,0.2,0.8\right\rangle,\left\langle u_{3}, 0.9,0.5,0.7\right\rangle\right], } \\
& {\left[\left(\neg e_{1}, r, 0\right),\left\langle u_{1}, 0.6,0.7,0.4\right\rangle\right], } \\
& {\left[\left(\neg e_{2}, p, 0\right),\left\langle u_{1}, 0.3,0.2,0.4\right\rangle,\left\langle u_{2}, 0.3,0.1,0.7\right\rangle\right], } \\
& {\left[\left(\neg e_{2}, q, 0\right),\left\langle u_{3}, 0.2,0.4,0.3\right\rangle\right], } \\
& {\left.\left[\left(\neg e_{2}, r, 0\right),\left\langle u_{2}, 0.9,0.4,0.3\right\rangle\right]\right\} . }
\end{aligned}
$$

3.6. Definition: Empty or null neutrosophic soft expert set with respect to parameter. A neutrosophic soft expert set $(H, A)$ over the universe $U$ is termed to be empty or null neutrosophic soft expert set with respect to the parameter $A$ if

$$
T_{H(e)}(m)=0, \quad F_{H(e)}(m)=0, \quad I_{H(e)}(m)=0, \quad \forall m \in U, \quad \forall e \in A .
$$

In this case the null neutrosophic soft expert set (NNSES) is denoted by $\Phi_{v_{A}}$.

3.5. Example: Let $U=\left\{u_{1}, u_{2}, u_{3}\right\}$ the set of three cars be considered as universal set $E=\{\operatorname{good}\}=\left\{e_{1}\right\}$ be the set of parameters that characterizes the car and let $X=\{p, q\}$ be a set of experts.

$$
\begin{aligned}
\Phi_{\triangleleft}= & (\text { NNSES }) \\
= & \left\{\left[\left(e_{1}, p, 1\right),\left\langle u_{1}, 0,0,0\right\rangle,\left\langle u_{2}, 0,0,0\right\rangle\right],\left[\left(e_{1}, q, 1\right),\left\langle u_{1}, 0,0,0\right\rangle,\left\langle u_{2}, 0,0,0\right\rangle\right],\left[\left(e_{1}, p, 0\right),\left\langle u_{3}, 0,0,0\right\rangle\right],\right. \\
& {\left.\left[\left(e_{1}, q, 0\right),\left\langle u_{3}, 0,0,0\right\rangle\right]\right\} . }
\end{aligned}
$$

Here the (NNSES) $(H, A)$ is the null neutrosophic soft expert sets.

3.7. Definition: An agree-neutrosophic soft expert set $(F, A)_{1}$ over $U$ is a neutrosophic soft expert subset of $(F, A)$ defined as follow

$$
(F, A)_{1}=\left\{F_{1}(m): m \in E \times X \times\{1\}\right\} .
$$

3.6. Example: Consider 3.1. Example. Then the agree-neutrosophic soft expert set $(F, A)_{1}$ over $U$ is 


$$
\begin{aligned}
&(F, A)_{1}=\left\{\left[\left(e_{1}, p, 1\right),\left\langle u_{1}, 0.3,0.5,0.7\right\rangle,\left\langle u_{3}, 0.5,0.6,0.3\right\rangle\right],\left[\left(e_{1}, q, 1\right),\left\langle u_{2}, 0.8,0.2,0.3\right\rangle,\left\langle u_{3}, 0.9,0.5,0.7\right\rangle\right],\right. \\
& {\left[\left(e_{1}, r, 1\right),\left\langle u_{1}, 0.4,0.7,0.6\right\rangle\right],\left[\left(e_{2}, p, 1\right),\left\langle u_{1}, 0.4,0.2,0.3\right\rangle,\left\langle u_{2}, 0.7,0.1,0.3\right\rangle\right] } \\
& {\left.\left[\left(e_{2}, q, 1\right),\left\langle u_{3}, 0.3,0.4,0.2\right\rangle\right],\left[\left(e_{2}, r, 1\right),\left\langle u_{2}, 0.3,0.4,0.9\right\rangle\right]\right\} . }
\end{aligned}
$$

3.8. Definition: A disagree-neutrosophic soft expert set $(F, A)_{0}$ over $U$ is a neutrosophic soft expert subset of $(F, A)$ defined as follow

$$
(F, A)_{0}=\left\{F_{0}(m): m \in E \times X \times\{0\}\right\} .
$$

3.7. Example: Consider 3.1. Example. Then the disagree-neutrosophic soft expert set $(F, A)_{0}$ over $U$ is

$$
\begin{aligned}
&(F, A)_{0}=\left\{\left[\left(e_{1}, p, 0\right),\left\langle u_{2}, 0.5,0.2,0.3\right\rangle\right],\left[\left(e_{1}, q, 0\right),\left\langle u_{1}, 0.6,0.3,0.5\right\rangle\right],\right. \\
& {\left[\left(e_{1}, r, 0\right),\left\langle u_{2}, 0.7,0.6,0.4\right\rangle,\left\langle u_{3}, 0.9,0.5,0.7\right\rangle\right],\left[\left(e_{2}, p, 0\right),\left\langle u_{3}, 0.7,0.9,0.6\right\rangle\right], } \\
& {\left.\left[\left(e_{2}, q, 0\right),\left\langle u_{1}, 0.7,0.3,0.6\right\rangle,\left\langle u_{2}, 0.6,0.2,0.5\right\rangle\right],\left[\left(e_{2}, r, 0\right),\left\langle u_{1}, 0.6,0.2,0.5\right\rangle,\left\langle u_{3}, 0.7,0.2,0.8\right\rangle\right]\right\} . }
\end{aligned}
$$

3.9. Definition: Union of two neutrosophic soft expert sets.

Let $(H, A)$ and $(G, B)$ be two NSESs over the common universe $U$. Then the union of $(H, A)$ and $(G, B)$ is denoted by " $(H, A) \tilde{\cup}(G, B)$ " and is defined by $(H, A) \tilde{\cup}(G, B)=(K, C)$, where $C=A \cup B$ and the truthmembership, indeterminacy-membership and falsity-membership of $(K, C)$ are as follows:

$$
\begin{gathered}
T_{H(e)}(m)= \begin{cases}T_{H(e)}(m), & \text { if } e \in A-B, \\
T_{G(e)}(m), & \text { if } e \in B-A, \\
\max \left(T_{H(e)}(m), T_{G(e)}(m)\right), & \text { if } e \in A \cap B .\end{cases} \\
I_{K(e)}(m)= \begin{cases}I_{H(e)}(m), & \text { if } e \in A-B, \\
I_{G(e)}(m), & \text { if } e \in B-A, \\
\left(I_{H(e)}(m), I_{G(e)}(m)\right), & \text { if } e \in A \cap B .\end{cases} \\
F_{H(e)}(m)= \begin{cases}F_{H(e)}(m), & \text { if } e \in A-B, \\
F_{G(e)}(m), & \text { if } e \in B-A, \\
\min \left(F_{H(e)}(m), F_{G(e)}(m)\right), & \text { if } e \in A \cap B .\end{cases}
\end{gathered}
$$

3.8. Example: Let $(H, A)$ and $(G, B)$ be two NSESs over the common universe $U$

$$
\begin{aligned}
& (H, A)=\left\{\left[\left(e_{1}, p, 1\right),\left\langle u_{1}, 0.3,0.5,0.7\right\rangle,\left\langle u_{3}, 0.5,0.6,0.2\right\rangle\right],\left[\left(e_{1}, q, 1\right),\left\langle u_{1}, 0.6,0.3,0.5\right\rangle,\left\langle u_{2}, 0.8,0.2,0.3\right\rangle\right]\right\}, \\
& (G, B)=\left\{\left(e_{1}, p, 1\right),\left\langle u_{1}, 0.4,0.6,0.2\right\rangle,\left\langle u_{2}, 0.7,0.5,0.8\right\rangle\right\} .
\end{aligned}
$$

Therefore $(H, A) \tilde{\cup}(G, B)=(K, C)$

$$
\begin{aligned}
&(K, C)=\left\{\left[\left(e_{1}, p, 1\right),\left\langle u_{1}, 0.4,0.55,0.2\right\rangle,\left\langle u_{2}, 0.7,0.5,0.8\right\rangle,\left\langle u_{3}, 0.5,0.6,0.2\right\rangle\right],\right. \\
& {\left.\left[\left(e_{1}, q, 1\right),\left\langle u_{1}, 0.6,0.3,0.5\right\rangle,\left\langle u_{2}, 0.8,0.2,0.3\right\rangle\right]\right\} . }
\end{aligned}
$$


3.10. Definition: Intersection of two neutrosophic soft expert sets. Let $(H, A)$ and $(G, B)$ be two NSESs over the common universe $U$. Then the intersection of $(H, A)$ and $(G, B)$ is denoted by " $(H, A) \tilde{\cap}(G, B)$ " and is defined by $(H, A) \tilde{\cap}(G, B)=(K, C)$, where $C=A \cap B$ and the truth-membership, indeterminacy-membership and falsity-membership of $(K, C)$ are as follows:

$$
\begin{aligned}
& T_{H(e)}(m)=\min \left(T_{H(e)}(m), T_{G(e)}(m)\right), \\
& I_{K(e)}(m)=\frac{I_{H(e)}(m)+I_{G(e)}(m)}{2}, \\
& F_{H(e)}(m)=\max \left(F_{H(e)}(m), F_{G(e)}(m)\right), \text { if } e \in A \cap B .
\end{aligned}
$$

3.9. Example: Let $(H, A)$ and $(G, B)$ be two NSESs over the common universe $U$

$$
\begin{aligned}
& (H, A)=\left\{\left[\left(e_{1}, p, 1\right),\left\langle u_{1}, 0.3,0.5,0.7\right\rangle,\left\langle u_{3}, 0.5,0.6,0.2\right\rangle\right],\left[\left(e_{1}, q, 1\right),\left\langle u_{1}, 0.6,0.3,0.5\right\rangle,\left\langle u_{2}, 0.8,0.2,0.3\right\rangle\right]\right\}, \\
& (G, B)=\left\{\left(e_{1}, p, 1\right),\left\langle u_{1}, 0.4,0.6,0.2\right\rangle,\left\langle u_{2}, 0.7,0.5,0.8\right\rangle\right\} .
\end{aligned}
$$

Therefore $(H, A) \tilde{\cap}(G, B)=(K, C)$

$$
(K, C)=\left\{\left(e_{1}, p, 1\right),\left\langle u_{1}, 0.3,0.55,0.7\right\rangle\right\} .
$$

3.1. Proposition: If $(H, A)$ and $(G, B)$ are neutrosophic soft expert sets over $U$. Then

1) $(H, A) \tilde{\cup}(G, B)=(G, B) \tilde{\cup}(H, A)$

2) $(H, A) \tilde{\cap}(G, B)=(G, B) \tilde{\cap}(H, A)$

3) $\left((H, A)^{c}\right)^{c}=(H, A)$

4) $(H, A) \tilde{\cup} \Phi=(H, A)$

5) $(H, A) \tilde{\cap} \Phi=\Phi$

Proof: 1 ) We want to prove that $(H, A) \tilde{\cup}(G, B)=(G, B) \tilde{\cup}(H, A)$ by using 3.9 definition and we consider the case when if $e \in A \cap B$ as the other cases are trivial, then we have

$$
\begin{aligned}
(H, A) \tilde{\cup}(G, B) & =\left\{\left\langle u, \max \left(T_{H(e)}(m), T_{G(e)}(m)\right), \frac{I_{H(e)}(m)+I_{G(e)}(m)}{2}, \min \left(F_{H(e)}(m), F_{G(e)}(m)\right)\right\rangle: u \in U\right\} \\
& =\left\{\left\langle u, \max \left(T_{G(e)}(m), T_{H(e)}(m)\right), \frac{I_{G(e)}(m)+I_{H(e)}(m)}{2}, \min \left(F_{G(e)}(m), F_{H(e)}(m)\right)\right\rangle: u \in U\right\} \\
& =(G, B) \tilde{\cup}(H, A) .
\end{aligned}
$$

The proof of the propositions 2) to 5) are obvious.

3.2. Proposition: If $(H, A),(G, B)$ and $(K, D)$ are three neutrosophic soft expert sets over $U$. Then

1) $((H, A) \tilde{\cup}(G, B)) \tilde{\cup}(M, D)=(H, A) \tilde{\cup}((G, B) \tilde{\cup}(M, D))$

2) $((H, A) \tilde{\cap}(G, B)) \tilde{\cap}(M, D)=(H, A) \tilde{\cap}((G, B) \tilde{\cap}(M, D))$

Proof: 1) We want to prove that $((H, A) \tilde{\cup}(G, B)) \tilde{\cup}(M, D)=(H, A) \tilde{\cup}((G, B) \tilde{\cup}(M, D))$ by using 3.9 definition and we consider the case when if $e \in A \cap B$ as the other cases are trivial, then we have

$$
(H, A) \tilde{\cup}(G, B)=\left\{\left\langle u, \max \left(T_{H(e)}(m), T_{G(e)}(m)\right), \frac{I_{H(e)}(m)+I_{G(e)}(m)}{2}, \min \left(F_{H(e)}(m), F_{G(e)}(m)\right)\right\rangle: u \in U\right\}
$$

We also consider her the case when $e \in D$ as the other cases are trivial, then we have 


$$
\begin{aligned}
& ((H, A) \tilde{\cup}(G, B)) \tilde{\cup}(M, D) \\
& =\left\{\left(\left\langle u, \max \left(T_{H(e)}(m), T_{G(e)}(m)\right), \frac{I_{H(e)}(m)+I_{G(e)}(m)}{2}, \min \left(F_{H(e)}(m), F_{G(e)}(m)\right)\right\rangle\right),\right. \\
& \left.\quad\left(T_{M(e)}(m), I_{M(e)}(m), F_{M(e)}(m)\right): u \in U\right\} \\
& =\left\{\left(T_{H(e)}(m), I_{H(e)}(m), F_{H(e)}(m)\right),\right. \\
& \left.\quad\left(\left\langle u, \max \left(T_{G(e)}(m), T_{M(e)}(m)\right), \frac{I_{G(e)}(m)+I_{M(e)}(m)}{2}, \min \left(F_{G(e)}(m), F_{M(e)}(m)\right)\right\rangle\right),: u \in U\right\} \\
& =(H, A) \tilde{\cup}((G, B) \tilde{\cup}(M, D)) .
\end{aligned}
$$

2) The proof is straightforward.

3.3. Proposition: If $(H, A),(G, B)$ and $(M, D)$ are three neutrosophic soft expert sets over $U$. Then

1) $((H, A) \tilde{\cup}(G, B)) \tilde{\cap}(M, D)=((H, A) \tilde{\cap}(M, D)) \tilde{\cup}((G, B) \tilde{\cap}(M, D))$

2) $((H, A) \tilde{\cap}(G, B)) \tilde{\cup}(M, D)=((H, A) \tilde{\cup}(M, D)) \tilde{\cap}((G, B) \tilde{\cup}(M, D))$

Proof: We use the same method as in the previous proof.

3.11. Definition: AND operation on two neutrosophic soft expert sets. Let $(H, A)$ and $(G, B)$ be two NSESs over the common universe $U$. Then "AND" operation on them is denoted by " $(H, A) \tilde{\wedge}(G, B)$ " and is defined by $(H, A) \tilde{\wedge}(G, B)=(K, A \times B)$ where the truth-membership, indeterminacy-membership and falsity-membership of $(K, A \times B)$ are as follows:

$$
\begin{aligned}
& T_{H(\alpha, \beta)}(m)=\min \left(T_{H(\alpha)}(m), T_{G(\beta)}(m)\right), \\
& I_{K(\alpha, \beta)}(m)=\frac{I_{H(\alpha)}(m)+I_{G(\beta)}(m)}{2}, \\
& F_{H(\alpha, \beta)}(m)=\max \left(F_{H(\alpha)}(m), F_{G(\beta)}(m)\right), \quad \text { if } \forall \alpha \in A, \forall \beta \in B .
\end{aligned}
$$

3.10. Example: Let $(H, A)$ and $(G, B)$ be two NSESs over the common universe $U$. Then $(H, A)$ and $(G, B)$ is a follows:

$$
\begin{aligned}
& (H, A)=\left\{\left[\left(e_{1}, p, 1\right),\left\langle u_{1}, 0.3,0.5,0.7\right\rangle,\left\langle u_{3}, 0.5,0.6,0.2\right\rangle\right],\left[\left(e_{1}, q, 1\right),\left\langle u_{1}, 0.6,0.3,0.5\right\rangle,\left\langle u_{2}, 0.8,0.2,0.3\right\rangle\right]\right\}, \\
& (G, B)=\left\{\left(e_{1}, p, 1\right),\left\langle u_{1}, 0.4,0.6,0.2\right\rangle,\left\langle u_{2}, 0.7,0.5,0.8\right\rangle\right\} .
\end{aligned}
$$

Therefore $(H, A) \tilde{\wedge}(G, B)=(K, A \times B)$

$$
\begin{aligned}
&(K, A \times B)=\left\{\left[\left(e_{1}, p, 1\right),\left(e_{1}, p, 1\right)\left\langle u_{1}, 0.3,0.55,0.7\right\rangle,\left\langle u_{2}, 0.7,0.5,0.8\right\rangle,\left\langle u_{3}, 0.5,0.6,0.2\right\rangle\right],\right. \\
& {\left.\left[\left(e_{1}, q, 1\right),\left(e_{1}, p, 1\right)\left\langle u_{1}, 0.4,0.45,0.5\right\rangle,\left\langle u_{2}, 0.7,0.35,0.8\right\rangle\right]\right\}, }
\end{aligned}
$$

3.12. Definition: OR operation on two neutrosophic soft expert sets. Let $(H, A)$ and $(G, B)$ be two NSESs over the common universe $U$. Then "OR" operation on them is denoted by “ $(H, A) \tilde{\vee}(G, B)$ " and is defined by $(H, A) \tilde{\vee}(G, B)=(O, A \times B)$ where the truth-membership, indeterminacy-membership and falsity-membership of $(O, A \times B)$ are as follows: 


$$
\begin{aligned}
& T_{O(\alpha, \beta)}(m)=\max \left(T_{H(\alpha)}(m), T_{G(\beta)}(m)\right), \\
& I_{O(\alpha, \beta)}(m)=\frac{I_{H(\alpha)}(m)+I_{G(\beta)}(m)}{2}, \\
& F_{O(\alpha, \beta)}(m)=\min \left(F_{H(\alpha)}(m), F_{G(\beta)}(m)\right), \text { if } \forall \alpha \in A, \forall \beta \in B .
\end{aligned}
$$

3.11. Example: Let $(H, A)$ and $(G, B)$ be two NSESs over the common universe $U$. Then $(H, A)$ OR $(G, B)$ is a follows:

$$
\begin{aligned}
& (H, A)=\left\{\left[\left(e_{1}, p, 1\right),\left\langle u_{1}, 0.3,0.5,0.7\right\rangle,\left\langle u_{3}, 0.5,0.6,0.2\right\rangle\right],\left[\left(e_{1}, q, 1\right),\left\langle u_{1}, 0.6,0.3,0.5\right\rangle\left\langle u_{2}, 0.8,0.2,0.3\right\rangle\right]\right\}, \\
& (G, B)=\left\{\left(e_{1}, p, 1\right),\left\langle u_{1}, 0.4,0.6,0.2\right\rangle,\left\langle u_{2}, 0.7,0.5,0.8\right\rangle\right\} .
\end{aligned}
$$

Therefore $(H, A) \tilde{v}(G, B)=(O, A \times B)$

$$
\begin{aligned}
&(O, A \times B)=\left\{\left[\left(e_{1}, p, 1\right),\left(e_{1}, p, 1\right)\left\langle u_{1}, 0.4,0.55,0.2\right\rangle,\left\langle u_{2}, 0.8,0.2,0.3\right\rangle,\left\langle u_{3}, 0.5,0.6,0.2\right\rangle\right],\right. \\
& {\left.\left[\left(e_{1}, q, 1\right),\left(e_{1}, p, 1\right)\left\langle u_{1}, 0.6,0.45,0.2\right\rangle,\left\langle u_{2}, 0.8,0.35,0.2\right\rangle,\left\langle u_{3}, 0.5,0.6,0.2\right\rangle\right]\right\} . }
\end{aligned}
$$

3.4. Proposition: If $(H, A)$ and $(G, B)$ are neutrosophic soft expert sets over $U$. Then

1) $((H, A) \tilde{\wedge}(G, B))^{c}=(H, A)^{c} \tilde{v}(G, B)^{c}$

2) $((H, A) \tilde{\vee}(G, B))^{c}=(H, A)^{c} \tilde{\wedge}(G, B)^{c}$

Proof: 1) Let $(H, A)=\left\{\left\langle u, T_{H(x)}(m), I_{H(x)}(m), F_{H(x)}(m)\right\rangle: u \in U\right\}$ and

$$
(G, B)=\left\{\left\langle u, T_{G(x)}(m), I_{G(x)}(m), F_{G(x)}(m)\right\rangle: u \in U\right\}
$$

be two NSESs over the common universe $U$. Also let $(H, A) \tilde{\wedge}(G, B)=(K, A \times B)$, where

$$
(K, A \times B)=\left\{\left\langle u, \min \left(T_{H(\alpha)}(m), T_{G(\beta)}(m)\right), \frac{I_{H(\alpha)}(m)+I_{G(\beta)}(m)}{2}, \max \left(F_{H(\alpha)}(m), F_{G(\beta)}(m)\right)\right\rangle: u \in U\right\}
$$

Therefore

$$
\begin{aligned}
((H, A) \tilde{\wedge}(G, B))^{c} & =(K, A \times B)^{c} \\
& =\left\{\left\langle u, \max \left(F_{H(\alpha)}(m), F_{G(\beta)}(m)\right), \frac{I_{H(\alpha)}(m)+I_{G(\beta)}(m)}{2}, \min \left(T_{H(\alpha)}(m), T_{G(\beta)}(m)\right)\right\rangle: u \in U\right\},
\end{aligned}
$$

Again

$$
\begin{aligned}
& (H, A)^{c} \tilde{v}(G, B)^{c} \\
& =\left\{\left\langle u, \max \left(F_{H^{c}(\alpha)}(m), F_{G^{c}(\beta)}(m)\right), \frac{I_{H^{c}(\alpha)}(m)+I_{G^{c}(\beta)}(m)}{2}, \min \left(T_{H^{c}(\alpha)}(m), T_{G^{c}(\beta)}(m)\right)\right\rangle: u \in U\right\} \\
& =\left\{\left\langle u, \min \left(T_{H(\alpha)}(m), T_{G(\beta)}(m)\right), \frac{I_{H(\alpha)}(m)+I_{G(\beta)}(m)}{2}, \max \left(F_{H(\alpha)}(m), F_{G(\beta)}(m)\right)\right\rangle: u \in U\right\} \\
& =\left\{\left\langle u, \max \left(F_{H(\alpha)}(m), F_{G(\beta)}(m)\right), \frac{I_{H(\alpha)}(m)+I_{G(\beta)}(m)}{2}, \min \left(T_{H(\alpha)}(m), T_{G(\beta)}(m)\right)\right\rangle: u \in U\right\} .
\end{aligned}
$$


Hence the result is proved.

3.5. Proposition: If $(H, A),(G, B)$ and $(M, D)$ are three neutrosophic soft expert sets over $U$. Then

1) $((H, A) \tilde{\vee}(G, B)) \tilde{\vee}(M, D)=(H, A) \tilde{\vee}((G, B) \tilde{\vee}(M, D))$

2) $((H, A) \tilde{\wedge}(G, B)) \tilde{\wedge}(M, D)=(H, A) \tilde{\wedge}((G, B) \tilde{\wedge}(M, D))$

3) $((H, A) \tilde{\vee}(G, B)) \tilde{\wedge}(M, D)=((H, A) \tilde{\wedge}(M, D)) \tilde{\vee}((G, B) \tilde{\wedge}(M, D))$

4) $((H, A) \tilde{\wedge}(G, B)) \tilde{\vee}(M, D)=((H, A) \tilde{\vee}(M, D)) \tilde{\wedge}((G, B) \tilde{\vee}(M, D))$

Proof: We use the same method as in the previous proof.

\section{An Application of Neutrosophic Soft Expert Set}

In this section, we present an application of neutrosophic soft expert set theory in a decision-making problem. The problem we consider is as below:

Suppose that a hospital to buy abed. Seven alternatives are as follows:

$$
U=\left\{u_{1}, u_{2}, u_{3}, u_{4}, u_{5}, u_{6}, u_{7}\right\},
$$

suppose there are five parameters $E=\left\{e_{1}, e_{2}, e_{3}, e_{4}, e_{5}\right\}$ where the parameters $e_{i}(i=1,2,3,4,5)$ stand for "medical bed", "soft bed", "orthopedic bed", "moving bed", "air bed", respectively. Let $X=\{p, q, r\}$ be a set of experts. Suppose:

$$
\begin{aligned}
(F, Z)=\{( & \left.\left(e_{1}, p, 1\right),\left\{u_{1}, u_{3}, u_{6}\right\}\right),\left(\left(e_{1}, q, 1\right),\left\{u_{1}, u_{3}, u_{4}, u_{7}\right\}\right), \\
& \left(\left(e_{1}, r, 1\right),\left\{u_{1}, u_{2}, u_{4}, u_{5}, u_{7}\right\}\right),\left(\left(e_{2}, p, 1\right),\left\{u_{3}, u_{5}, u_{6}, u_{7}\right\}\right), \\
& \left(\left(e_{2}, q, 1\right),\left\{u_{1}, u_{3}, u_{4}, u_{6}\right\}\right),\left(\left(e_{2}, r, 1\right),\left\{u_{1}, u_{3}, u_{4}, u_{5}\right\}\right), \\
& \left(\left(e_{3}, p, 1\right),\left\{u_{1}, u_{2}, u_{6}, u_{7}\right\}\right),\left(\left(e_{3}, q, 1\right),\left\{u_{1}, u_{2}, u_{4}, u_{5}, u_{7}\right\}\right), \\
& \left(\left(e_{3}, r, 1\right),\left\{u_{1}, u_{2}, u_{3}, u_{4}, u_{6}, u_{7}\right\}\right),\left(\left(e_{4}, p, 1\right),\left\{u_{1}, u_{2}, u_{5}, u_{6}\right\}\right), \\
& \left(\left(e_{4}, q, 1\right),\left\{u_{2}, u_{3}, u_{4}, u_{6}, u_{7}\right\}\right),\left(\left(e_{4}, r, 1\right),\left\{u_{1}, u_{2}, u_{3}, u_{5}, u_{6}\right\}\right), \\
& \left(\left(e_{5}, p, 1\right),\left\{u_{1}, u_{3}, u_{4}, u_{5}, u_{6}, u_{7}\right\}\right),\left(\left(e_{5}, q, 1\right),\left\{u_{3}, u_{4}, u_{5}\right\}\right), \\
& \left(\left(e_{5}, r, 1\right),\left\{u_{1}, u_{3}, u_{4}, u_{7}\right\}\right),\left(\left(e_{1}, p, 0\right),\left\{u_{2}, u_{4}, u_{5}, u_{7}\right\}\right), \\
& \left(\left(e_{1}, q, 0\right),\left\{u_{2}, u_{5}, u_{6}\right\}\right),\left(\left(e_{1}, r, 0\right),\left\{u_{3}, u_{6}\right\}\right), \\
& \left(\left(e_{2}, p, 0\right),\left\{u_{1}, u_{2}, u_{4}\right\}\right),\left(\left(e_{2}, q, 0\right),\left\{u_{2}, u_{5}, u_{7}\right\}\right), \\
& \left(\left(e_{2}, r, 0\right),\left\{u_{2}, u_{6}, u_{7}\right\}\right),\left(\left(e_{3}, p, 0\right),\left\{u_{3}, u_{4}, u_{5}\right\}\right),\left(\left(e_{3}, q, 0\right),\left\{u_{3}, u_{6}\right\}\right), \\
& \left(\left(e_{3}, r, 0\right),\left\{u_{5}\right\}\right),\left(\left(e_{4}, p, 0\right),\left\{u_{3}, u_{4}, u_{7}\right\}\right), \\
& \left(\left(e_{4}, q, 0\right),\left\{u_{1}, u_{5}\right\}\right),\left(\left(e_{4}, r, 0\right),\left\{u_{4}, u_{7}\right\}\right),\left(\left(e_{5}, p, 0\right),\left\{u_{2}\right\}\right), \\
& \left.\left(\left(e_{5}, q, 0\right),\left\{u_{1}, u_{2}, u_{6}, u_{7}\right\}\right),\left(\left(e_{5}, r, 0\right),\left\{u_{2}, u_{5}, u_{6}\right\}\right)\right\} .
\end{aligned}
$$

In Table 1 and Table 2 we present the agree-neutrosophic soft expert set and disagree-neutrosophic soft expert set, respectively, such that if $u_{i j} \in F_{1}(\varepsilon)$ then $u_{i j}=1$ otherwise $u_{i j}=0$, and if $u_{i j} \in F_{0}(\varepsilon)$ then $u_{i j}=1$ otherwise $u_{i j}=0$ where $u_{i j}$ are the entries in Table 1 and Table 2.

The following algorithm may be followed by the hospital wants to buy a bed.

1) input the neutrosophic soft expert set $(F, Z)$,

2) find an agree-neutrosophic soft expert set and a disagree-soft expert set,

3) find $c_{j}=\sum_{i} u_{i j}$ for agree-neutrosophic soft expert set,

4) find $k_{j}=\sum_{i} u_{i j}$ for disagree-neutrosophic soft expert set,

5) find $s_{j}=c_{j}-k_{j}$,

6) find $m$, for which $s_{m}=\operatorname{maxs}_{j}$. 
Table 1. Agree-neutrosophic soft expert set.

\begin{tabular}{|c|c|c|c|c|c|c|c|}
\hline$U$ & $u_{1}$ & $u_{2}$ & $u_{3}$ & $u_{4}$ & $u_{5}$ & $u_{6}$ & $u_{7}$ \\
\hline$\left(e_{1}, p\right)$ & 1 & 0 & 1 & 0 & 0 & 1 & 0 \\
\hline$\left(e_{2}, p\right)$ & 0 & 0 & 1 & 0 & 1 & 1 & 1 \\
\hline$\left(e_{3}, p\right)$ & 1 & 1 & 0 & 0 & 0 & 1 & 1 \\
\hline$\left(e_{4}, p\right)$ & 1 & 1 & 0 & 0 & 1 & 1 & 0 \\
\hline$\left(e_{5}, p\right)$ & 1 & 0 & 1 & 1 & 1 & 1 & 1 \\
\hline$\left(e_{1}, q\right)$ & 1 & 0 & 1 & 1 & 0 & 0 & 1 \\
\hline$\left(e_{2}, q\right)$ & 1 & 0 & 1 & 1 & 0 & 1 & 0 \\
\hline$\left(e_{3}, q\right)$ & 1 & 1 & 0 & 1 & 1 & 0 & 1 \\
\hline$\left(e_{4}, q\right)$ & 0 & 1 & 1 & 1 & 0 & 1 & 1 \\
\hline$\left(e_{5}, q\right)$ & 0 & 0 & 1 & 1 & 1 & 0 & 0 \\
\hline$\left(e_{1}, r\right)$ & 1 & 1 & 0 & 1 & 1 & 0 & 1 \\
\hline$\left(e_{2}, r\right)$ & 1 & 0 & 1 & 1 & 1 & 0 & 0 \\
\hline$\left(e_{3}, r\right)$ & 1 & 1 & 1 & 1 & 0 & 1 & 1 \\
\hline$\left(e_{4}, r\right)$ & 1 & 1 & 1 & 0 & 1 & 1 & 0 \\
\hline$\left(e_{5}, r\right)$ & 1 & 0 & 1 & 1 & 0 & 0 & 1 \\
\hline$c_{j}=\sum_{i} u_{i j}$ & $c_{1}=12$ & $C_{2}=7$ & $c_{3}=11$ & $C_{4}=10$ & $C_{5}=7$ & $C_{6}=9$ & $c_{7}=9$ \\
\hline
\end{tabular}

Table 2. Disagree-neutrosophic soft expert set.

\begin{tabular}{|c|c|c|c|c|c|c|c|}
\hline$U$ & $u_{1}$ & $u_{2}$ & $u_{3}$ & $u_{4}$ & $u_{5}$ & $u_{6}$ & $u_{7}$ \\
\hline$\left(e_{1}, p\right)$ & 0 & 1 & 0 & 1 & 1 & 0 & 1 \\
\hline$\left(e_{2}, p\right)$ & 1 & 1 & 0 & 1 & 0 & 0 & 0 \\
\hline$\left(e_{3}, p\right)$ & 0 & 0 & 1 & 1 & 1 & 0 & 0 \\
\hline$\left(e_{4}, p\right)$ & 0 & 0 & 1 & 1 & 0 & 0 & 1 \\
\hline$\left(e_{5}, p\right)$ & 0 & 1 & 0 & 0 & 0 & 0 & 0 \\
\hline$\left(e_{1}, q\right)$ & 0 & 1 & 0 & 0 & 1 & 1 & 0 \\
\hline$\left(e_{2}, q\right)$ & 0 & 1 & 0 & 0 & 1 & 0 & 1 \\
\hline$\left(e_{3}, q\right)$ & 0 & 0 & 1 & 0 & 0 & 1 & 0 \\
\hline$\left(e_{4}, q\right)$ & 1 & 0 & 0 & 0 & 1 & 0 & 0 \\
\hline$\left(e_{5}, q\right)$ & 1 & 1 & 0 & 0 & 0 & 1 & 1 \\
\hline$\left(e_{1}, r\right)$ & 0 & 0 & 1 & 0 & 0 & 1 & 0 \\
\hline$\left(e_{2}, r\right)$ & 0 & 1 & 0 & 0 & 0 & 1 & 1 \\
\hline$\left(e_{3}, r\right)$ & 0 & 0 & 0 & 0 & 1 & 0 & 0 \\
\hline$\left(e_{4}, r\right)$ & 0 & 0 & 0 & 1 & 0 & 0 & 1 \\
\hline$\left(e_{5}, r\right)$ & 0 & 1 & 0 & 0 & 1 & 1 & 0 \\
\hline$k_{j}=\sum_{i} u_{i j}$ & $k_{1}=3$ & $k_{2}=8$ & $k_{3}=4$ & $k_{4}=5$ & $k_{5}=7$ & $k_{6}=6$ & $k_{7}=6$ \\
\hline
\end{tabular}


Table 3. $s_{j}=c_{j}-k_{j}$.

\begin{tabular}{ccc}
\hline$c_{j}=\sum_{i} u_{i j}$ & $k_{j}=\sum_{i} u_{i j}$ & $s_{j}=c_{j}-k_{j}$ \\
$c_{1}=12$ & $k_{1}=3$ & $s_{1}=9$ \\
$c_{2}=7$ & $k_{2}=8$ & $s_{2}=-1$ \\
$c_{3}=11$ & $k_{3}=4$ & $s_{3}=7$ \\
$c_{4}=10$ & $k_{4}=5$ & $s_{4}=5$ \\
$c_{5}=7$ & $k_{5}=7$ & $s_{5}=0$ \\
$c_{6}=9$ & $k_{6}=6$ & $s_{6}=3$ \\
$c_{7}=9$ & $k_{7}=6$ & $s_{7}=3$ \\
\hline
\end{tabular}

Then $s_{m}$ is the optimal choice object. If $m$ has more than one value, then any one of them could be chosen by hospital using its option. Now we use this algorithm to find the best choices for to get to the hospital bed. From Table 1 and Table 2 we have Table 3.

Then $\operatorname{maxs}_{j}=s_{1}$, so the hospital will select the bed $u_{1}$. In any case if they do not want to choose $u_{1}$ due to some reasons they second choice will be $u_{3}$.

\section{Conclusion}

In this paper, we have introduced the concept of neutrosophic soft expert set which is more effective and useful and studied some of its properties. Also the basic operations on neutrosophic soft expert set namely complement, union, intersection, AND and OR have been defined. Finally, we have presented an application of NSES in a decision-making problem.

\section{References}

[1] Atanassov, K. (1986) Intuitionistic Fuzzy Sets. Fuzzy Sets and Systems, 20, 87-96. http://dx.doi.org/10.1016/S0165-0114(86)80034-3

[2] Smarandache, F. (2005) Neutrosophic Set, a Generalization of the Intuitionistic Fuzzy Sets. International Journal of Pure and Applied Mathematics, 24, 287-297.

[3] Smarandache, F. (1998) A Unifying Field in Logics. Neutrosophy: Neutrosophic Probability, Set and Logic. American Research Press, Rehoboth.

[4] Molodtsov, D.A. (1999) Soft Set Theory-First Results. Computers and Mathematics with Applications, 37, $19-31$. http://dx.doi.org/10.1016/S0898-1221(99)00056-5

[5] Chen, D., Tsang, E.C.C., Yeung, D.S. and Wang, X. (2005) The Parameterization Reduction of Soft Sets and Its Application. Computers and Mathematics with Applications, 49, 757-763. http://dx.doi.org/10.1016/j.camwa.2004.10.036

[6] Maji, P.K., Roy, A.R. and Biswas, R. (2003) Soft Set Theory. Computers and Mathematics with Applications, 45, 555562. http://dx.doi.org/10.1016/S0898-1221(03)00016-6

[7] Maji, P.K. (2013) Neutrosophic Soft Set. Computers and Mathematics with Applications, 45, 555-562. http://dx.doi.org/10.1016/S0898-1221(03)00016-6

[8] Alkhazaleh, S., Salleh, A.R. and Hassan, N. (2011) Possibility Fuzzy Soft Set. Advances in Decision Sciences, 2011, Article ID: 479756. http://dx.doi.org/10.1155/2011/479756

[9] Alkhazaleh, S., Salleh, A.R. and Hassan, N. (2011) Fuzzy Parameterized Interval-Valued Fuzzy Soft Set. Applied Mathematical Sciences, 5, 3335-3346.

[10] Alkhazaleh, S. and Salleh, A.R. (2011) Soft Expert Sets. Advances in Decision Sciences, 2011, Article ID: 757868. http://dx.doi.org/10.1155/2011/757868

[11] Alkhazaleh, S. and Salleh, A.R. (2014) Fuzzy Soft Expert Set and Its Application. Applied Mathematics, 5, $1349-1368$. http://dx.doi.org/10.4236/am.2014.59127 
Scientific Research Publishing (SCIRP) is one of the largest Open Access journal publishers. It is currently publishing more than 200 open access, online, peer-reviewed journals covering a wide range of academic disciplines. SCIRP serves the worldwide academic communities and contributes to the progress and application of science with its publication.

Other selected journals from SCIRP are listed as below. Submit your manuscript to us via either submit@scirp.org or Online Submission Portal.
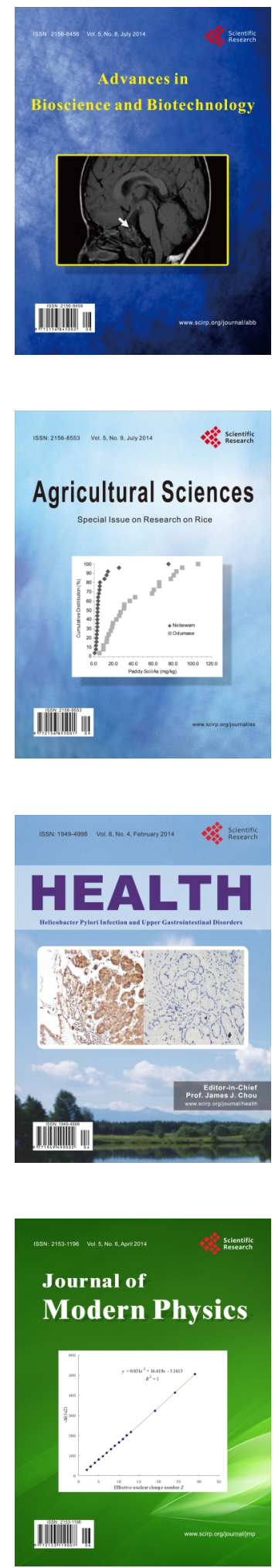
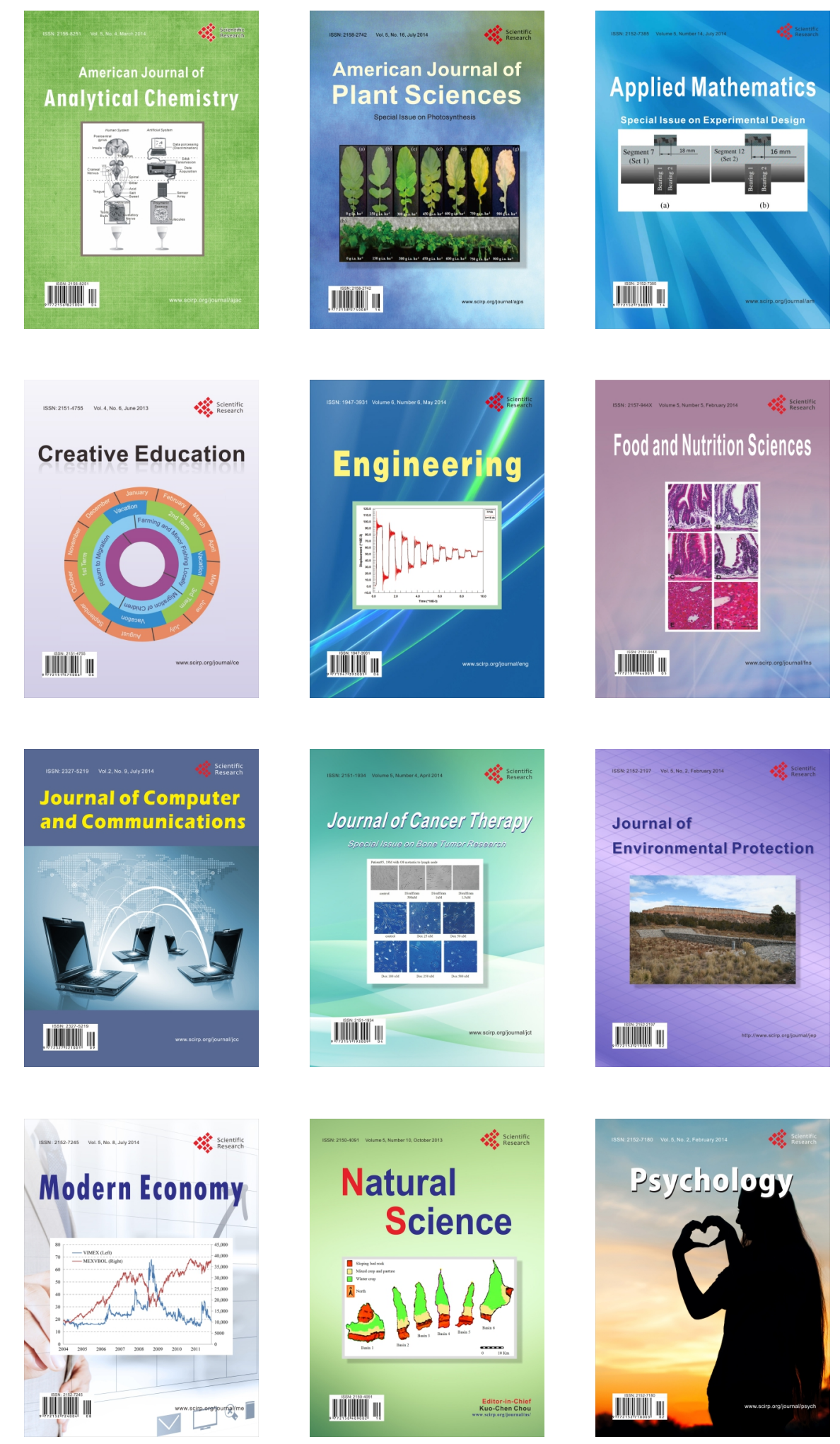